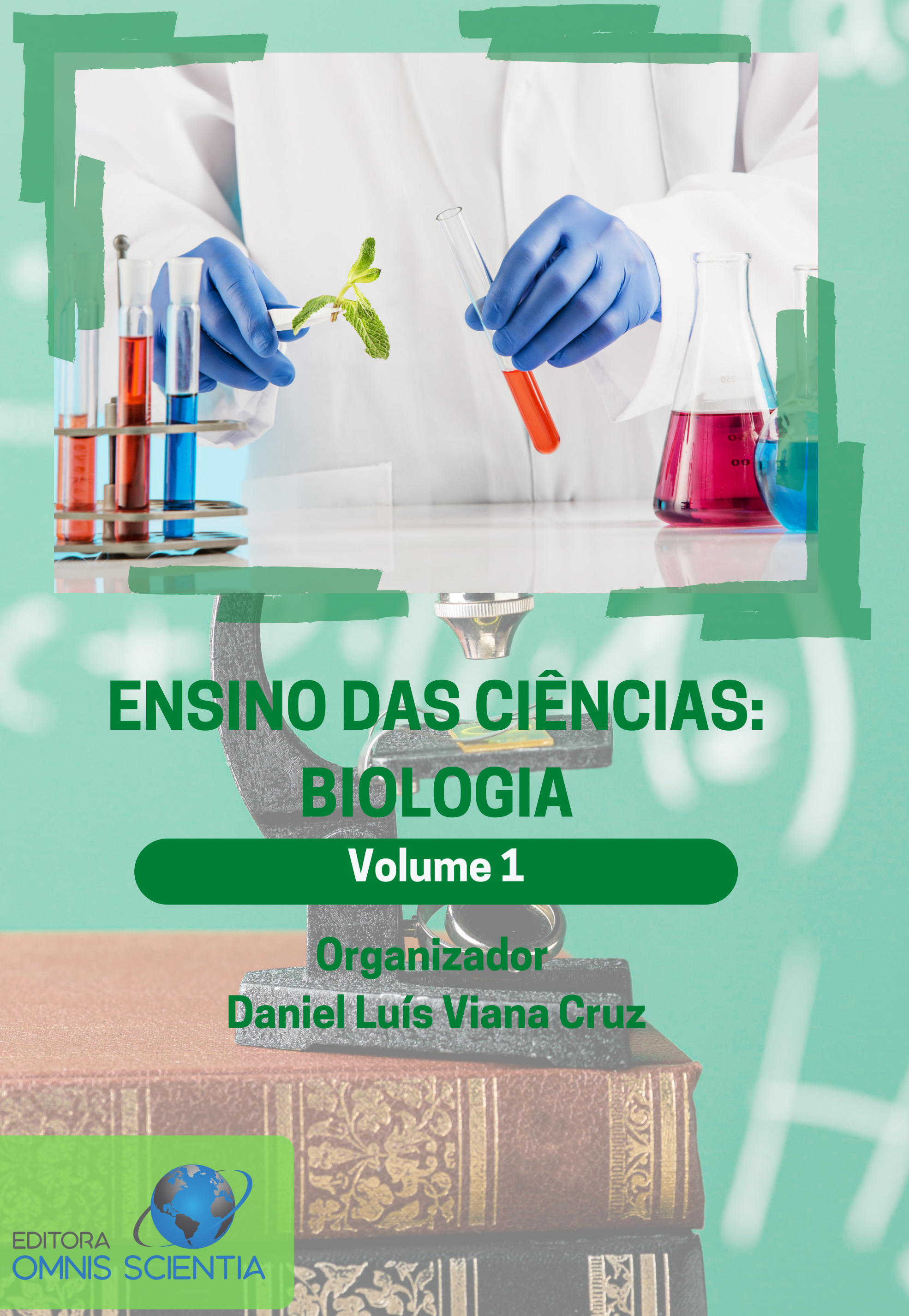




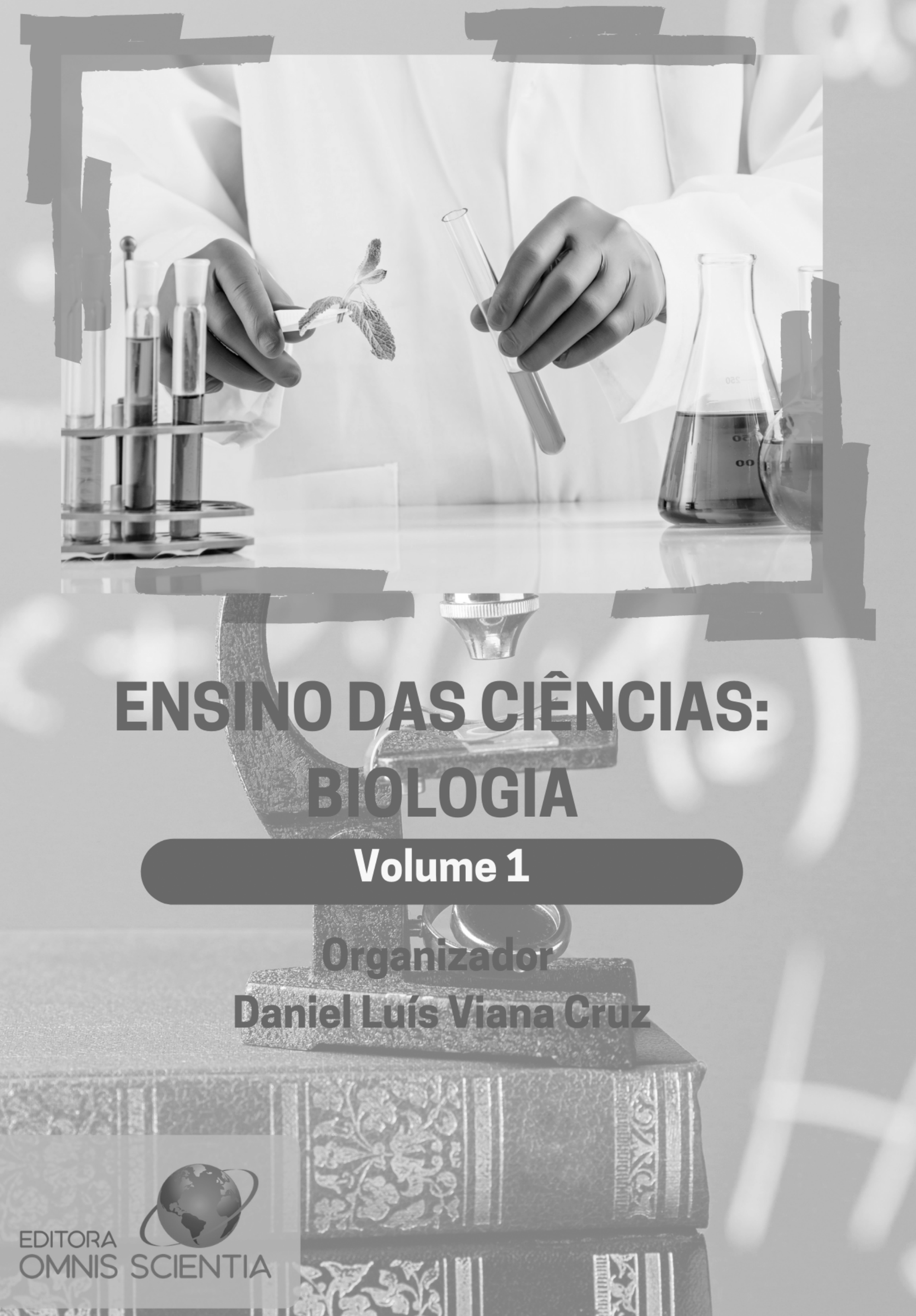


Editora Omnis Scientia

\section{ENSINO DAS CIÊNCIAS: BIOLOGIA}

Volume 1

$1^{\mathrm{a}}$ Edição

TRIUNFO - PE 


\section{Editor-Chefe}

Me. Daniel Luís Viana Cruz

Organizador (a)

Me. Daniel Luís Viana Cruz

\section{Conselho Editorial}

Dra. Pauliana Valéria Machado Galvão

Dr. Wendel José Teles Pontes

Dr. Walter Santos Evangelista Júnior

Dr. Cássio Brancaleone

Dr. Plínio Pereira Gomes Júnior

Editores de Área - Ciências Humanas

Dr. Cássio Brancaleone

Dr. José Edvânio da Silva

Assistentes Editoriais

Thialla Larangeira Amorim

Andrea Telino Gomes

Imagem de Capa

Freepik

\section{Edição de Arte}

Leandro José Dionísio

Revisão

Os autores

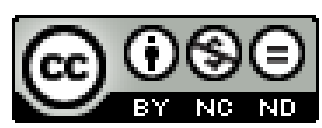

Este trabalho está licenciado com uma Licença Creative Commons - AtribuiçãoNãoComercial-SemDerivações 4.0 Internacional.

O conteúdo abordado nos artigos, seus dados em sua forma, correção e confiabilidade são de responsabilidade exclusiva dos autores. 


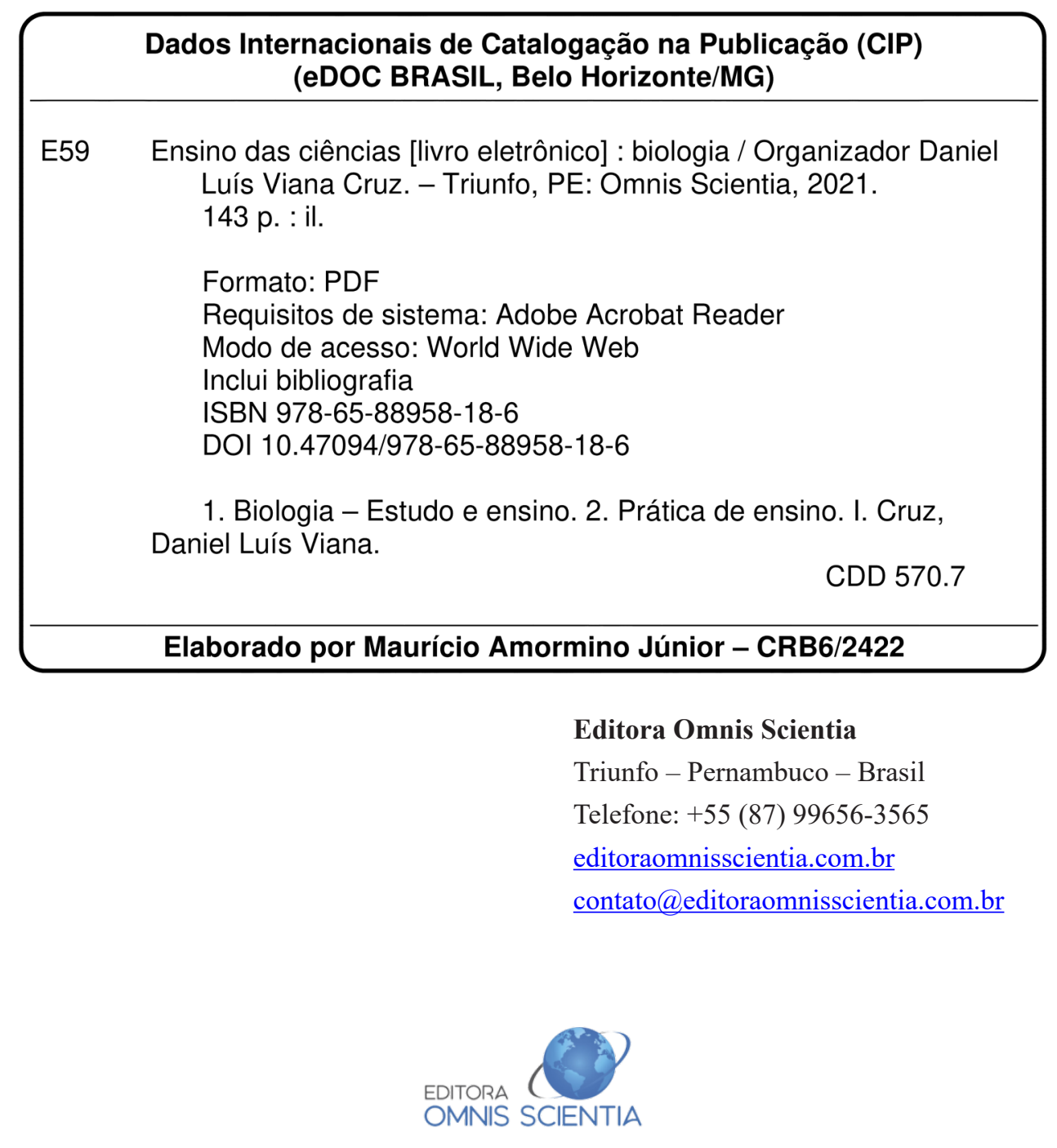




\section{PREFÁCIO}

O ensino é mais do que uma vocação, no país que vivemos trata-se de um sacerdócio. Ensinar, com poucos recursos e sem o merecido reconhecimento por parte da sociedade é persistir numa luta sem fim. Principalmente nesse período obscurecido pela desinformação e pelo negacionismo. Mas quando falamos de ensinar ciências, isso se torna ainda mais complexo, pois poucas escolas, sejam elas públicas ou privadas, possuem infraestrutura para aulas práticas. Que são tão importantes na fixação da informação. E assim os professores das ciências (Matemática, Física, Química e Biologia) seguem fazendo "mágica" nas salas de aula para que os alunos aprendam o mínimo necessário para a vida. Quando se trata de ensina Biologia, há muito que fazer com poucos recursos e o mínimo de boa vontade. E nessa obra o leitor poderá se inspirar em metodologias e ideias muito interessantes publicadas pelos autores.

Em nossos livros selecionamos um dos capítulos para premiação como forma de incentivo para os autores, e entre os excelentes trabalhos selecionados para compor este livro, o premiado foi o capítulo 3, intitulado "UM OLHAR PARA O ENFRENTAMENTO DAS VISÕES INGÊNUAS SOBRE A CIÊNCIA NO ENSINO DE BIOLOGIA”. 


\section{SUMÁRIO}

CAPÍTULO 1

AULAS REMOTAS DE CIÊNCIAS E BIOLOGIA DURANTE A PANDEMIA DO COVID-19 NA PERSPECTIVA DOS DISCENTES

Priscila Chaves de Souza

Hélio da Guia Alves Junior

DOI: $10.47094 / 978-65-88958-18-6 / 11-18$

CAPÍTULO 2 19

PRODUÇÃO E UTILIZAÇÃO DE MATERIAIS DIDÁTICOS NO ENSINO - APRENDIZAGEM DE DOENÇAS PARASITÁRIAS COMO: AMEBÍASE.

Sarah Lorena Silva Santos

Talessa Viegas Araujo

Samara Alves Correa

Lara Vitória Ribeiro Ferreia

Suelen Rocha Botão Ferreira

Lise Maria Mendes Holanda de Melo Ferreira

DOI: 10.47094/978-65-88958-18-6/19-27

CAPÍTULO 3 .28

UM OLHAR PARA O ENFRENTAMENTO DAS VISÕES INGÊNUAS SOBRE A CIÊNCIA NO ENSINO DE BIOLOGIA

Elda Cristina Carneiro da Silva;

Joanez Aparecida Aires

DOI: 10.47094/978-65-88958-18-6/28-41 
CAPÍTULO 4

ABORDAGEM DO TEMA SISTEMAS DE ENTREGA DE FÁRMACOS NO ENSINO FUNDAMENTAL

Edmilson Clarindo de Siqueira

José Adonias Alves de França

Silvana Caroline de Holanda

DOI: $10.47094 / 978-65-88958-18-6 / 42-52$

CAPÍTULO 5

A NANOTECNOLOGIA APLICADA AO ENSINO DE CIÊNCIAS

Edmilson Clarindo de Siqueira

José Adonias Alves de França

Silvana Caroline de Holanda

Fábio Rocha Formiga

DOI: $10.47094 / 978-65-88958-18-6 / 53-64$

CAPÍTULO 6 .65

OFICINAS DE CORDEL COM TEMAS DE BIOLOGIA

Edmilson Clarindo de Siqueira

José Adonias Alves de França

Silvana Caroline de Holanda

DOI: $10.47094 / 978-65-88958-18-6 / 65-75$

CAPÍTULO 7.

RELAÇÃO HOMEM E NATUREZA NAS MARGENS DO RIO PERICUMÃ NA CIDADE DE PINHEIRO-MA

Gabrielly Soares Dias Gonçalves 
Jenilce Monica Ferreira Fernandes

Werberth Braga Bastos

Hellen José Daiane Alves Reis

DOI: $10.47094 / 978-65-88958-18-6 / 76-86$

CAPÍTULO 8 .87

METODOLOGIAS ATIVAS NO ENSINO DE FISIOLOGIAHUMANA: O OLHAR DOS FUTUROS PROFESSORES

Maria Iracema Barbosa Moura

Francisco de Assis Pereira da Silva

Helayne Barbosa Moura

DOI: 10.47094/978-65-88958-18-6/87-96

CAPÍTULO 9 97

CORRIDA DE ESPERMATOZOIDES: SEXO E HERANÇA - UMA PROPOSTA INTERATIVA PARA O ENSINO DE HEREDITARIEDADE

Fernanda Pacheco-Fernandes

Benn Richard Alle

Iris Hass

Luciane Viater Turek

Maíra Alexandre Peres

Lupe Furtado-Alle

DOI: $10.47094 / 978-65-88958-18-6 / 97-112$

CAPÍTULO 10 113

DANÇA DOS CROMOSSOMOS: USANDO A LUDICIDADE PARA ENSINAR HEREDITARIEDADE 
Fernanda Pacheco-Fernandes

Benn Richard Alle

Iris Hass

Luciane Viater Tureck

Maíra Alexandre Peres

Lupe Furtado-Alle

DOI: $10.47094 / 978-65-88958-18-6 / 113-126$

CAPÍTULO 1

A DiscuSSÃO DA HOMOSSEXUALIDADE EM UM LIVRO PARADIDÁTICO DE SEXUALIDADE

Lucas Mendes Silva

Vitoria Raquel Pereira de Souza

Jackson Ronie Sá-Silva

DOI: $10.47094 / 978-65-88958-18-6 / 127-137$ 


\title{
PRODUÇÃO E UTILIZAÇÃO DE MATERIAIS DIDÁTICOS NO ENSINO - APRENDIZAGEM DE DOENÇAS PARASITÁRIAS COMO: AMEBÍASE.
}

\author{
Sarah Lorena Silva Santos ${ }^{1}$ \\ Universidade Estadual do Maranhão - UEMA, Pinheiro, Maranhão. \\ http://lattes.cnpq.br/2104955499438799 \\ Talessa Viegas Araujo ${ }^{2}$ \\ Universidade Estadual do Maranhão - UEMA, Pinheiro, Maranhão. \\ http://lattes.cnpq.br/6831347735863642

\section{Samara Alves Correa ${ }^{3}$} \\ Universidade Estadual do Maranhão - UEMA, Pinheiro, Maranhão. \\ http://lattes.cnpq.br/8361717654605904
}

\section{Lara Vitória Ribeiro Ferreia ${ }^{4}$}

Universidade Estadual do Maranhão - UEMA, Pinheiro, Maranhão.

http://lattes.cnpq.br/7159969665737451

\section{Suelen Rocha Botão Ferreira ${ }^{5}$}

Universidade Estadual do Maranhão - UEMA, Pinheiro, Maranhão.

http://lattes.cnpq.br/1272233351902347

\section{Lise Maria Mendes Holanda de Melo Ferreira ${ }^{6}$ \\ Universidade Estadual do Maranhão - UEMA, Pinheiro, Maranhão. \\ http://lattes.cnpq.br/1268167537620626}

RESUMO: O ensino de parasitologia aborda temas com linguagem e conceitos complexos sobre doenças negligenciadas, o que dificulta no aprendizado dos temas propostos, principalmente em regiões onde existe um grande déficit informativo. Portanto é de fundamental importância à utilização de recursos didáticos alternativos para que haja um melhor aprendizado por meio de metodologias mais dinâmicas para auxiliar na compreensão e fixação das informações e consequentemente a 
disseminação de conhecimento aliando na prevenção e diminuição de ocorrências dessas doenças. O presente trabalho teve como objetivo facilitar o entendimento sobre a amebíase causada pelo protozoário Entamoeba histolytica, através da confecção de materiais didáticos e apresentação do mesmo durante o I Simpósio Pinheirense de Biologia (SIMPHOBIO, 2019). Foram produzidas maquetes 3D em biscuit abordando o modo de transmissão e ciclo da doença, reaproveitamento de manequim para identificação dos órgãos afetados pelo parasita. Além disso, a apresentação com jogo, banner autoexplicativo e panfletos informativos. Mediante a utilização dos materiais didáticos com os ouvintes que por ali passavam, percebeu-se que o assunto considerado complexo se tornou de fácil compreensão, pois, os recursos utilizados auxiliaram no processo de ensino-aprendizagem, atraindo a atenção e a curiosidade dos espectadores para o tema abordado. Através da utilização do banner e dos panfletos como material de apoio houve uma explicação mais completa ficando acessível para que pudessem ler, propiciando assim uma maior facilidade na compreensão do assunto abordado e entendimento sobre os métodos profiláticos. Após a aplicação dos recursos didáticos, ficou evidente que a utilização de materiais com baixo custo pode auxiliar no processo de aprendizagem tanto dos discentes quanto da comunidade em geral, podendo ser aplicado em diversos ambientes onde exista a necessidade de um conteúdo informativo mais dinâmico sobre a doença, pois, são bastante eficazes na mediação do processo de ensino-aprendizagem.

PALAVRAS-CHAVE: Doenças negligenciadas. Ensino. Parasitologia.

\title{
PRODUCTION AND USE OF TEACHING MATERIALS IN TEACHING - LEARNING OF PARASITIC DISEASES AS: AMEBIASIS.
}

\begin{abstract}
The teaching of parasitology addresses topics with complex language and concepts about neglected diseases, which makes it difficult to learn the proposed themes, especially in regions where there is a large information deficit. Therefore, it is of fundamental importance to use alternative teaching resources so that there is better learning through more dynamic methodologies to assist in the understanding and fixation of information and, consequently, the dissemination of knowledge, combining the prevention and reduction of occurrences of these diseases. The present work aimed to facilitate the understanding of amebiasis caused by the protozoan Entamoeba histolytica, through the preparation of teaching materials and presentation during the 1st Pinheirense Biology Symposium (SIMPHOBIO, 2019). 3D models were produced in biscuit addressing the mode of disease transmission and cycle, reuse of mannequin to identify the organs affected by the parasite. In addition, the presentation with game, self-explanatory banner and information brochures. Through the use of teaching materials for listeners who passed by, it was noticed that the subject that was considered complex became easy to understand, because the resources used helped in the teaching-learning process, attracting attention and the curiosity of the viewers for the topic addressed. Through the use of the banner and brochures as support material, there was a more complete explanation available for them to read, thus providing a greater ease in understanding the subject addressed and understanding
\end{abstract}


about prophylactic methods. After the application of didactic resources, it was evident that the use of low-cost materials can assist in the learning process of both students and the community in general, and can be applied in different environments where there is a need for more dynamic information content about the disease, therefore, they are quite effective in mediating the teaching-learning process.

KEY WORDS: Neglected diseases. Teaching. Parasitology.

\section{INTRODUÇÃO}

As doenças parasitárias, cujos agentes etiológicos podem ser helmintos e protozoários, acometem um alto percentual de pessoas no mundo, sendo endêmicas em países em desenvolvimento ou subdesenvolvidos, concentrando seus casos em populações que apresentam déficit em educação, más condições de habitação, abastecimento de água potável e saneamento básico precários, condições estas que favorecem a evolução e propagação destas doenças entre a população (CARVALHO-COSTA et al., 2007; BASSO et al., 2008). Essas doenças parasitárias representam um sério problema de saúde pública mundial e podem ser prevenidos por disseminação de conhecimento em educação em saúde (LIMA et al., 2016).

Além disso, uma grande problemática em relação a disciplina de parasitologia é a condução da disciplina de forma técnica, desenvolvida de maneira convencional nas universidades, através da exposição dos conteúdos nas aulas teóricas e, na execução de protocolos para o desenvolvimento de atividades relacionadas a esses conteúdos, nas aulas práticas. Este modelo não explora a motivação de graduandos e docentes a desenvolverem práticas investigativas e reflexivas e de compreensão facilitada que contemple a aprendizagem eficiente do conteúdo da disciplina a um maior número de pessoas, se esquecendo que a vivência de experiências no cotidiano da prática profissional demanda contribuições ao desenvolvimento acadêmico profissional dos discentes (TAVARES et al., 2007).

Já que esses acadêmicos são agentes multiplicadores e de transferência de conhecimento a população. Essa problemática na formação dos licenciados em ciências biológicas, ocasiona muita disparidade entre a teoria ofertada nas instituições formadoras e a prática que terá que ser exercida em sala de aula, muitas vezes em escolas públicas (NASCIMENTO et al., 2015; TESORI, 2018).

Dentro desse contexto, o ensino de Parasitologia também possui linguagem e conceitos complexos que muitas vezes são mal apresentados prejudicando a aprendizagem e contribuindo para o desinteresse por parte dos alunos de graduação, que serão provavelmente futuros professores em diferentes esferas o ensino, principalmente ensino fundamental e médio.

Dentre as doenças parasitárias acometidas pelos seres humanos, a amebíase é bem comum (LORENZETTI; DELIZOICOV, 2001). Vale ressaltar que a amebíase é uma infecção parasitária causada pelo protozoário Entamoeba histolytica, popularmente conhecida como ameba, este é altamente patogênico que parasita o intestino (ALMEIDA; LEITE, 2020). Sendo encontrada em todo o mundo, principalmente em países em desenvolvimento e onde a população é exposta a condições 
de baixo nível de saneamento básico (BARBOSA et al., 2009). A infecção no homem se dá após a ingestão da forma cística madura contida em alimentos, água ou por qualquer tipo de contato fecaloral (CORDEIRO; MACEDO, 2007).

Portanto, é de fundamental importância que haja educação em saúde deixando de lado práticas tradicionais de difícil aprendizagem e adotando novas dinâmicas de ensino facilitando a compreensão dos discentes e da comunidade em geral. Recursos didáticos são materiais utilizados como auxílio no ensino aprendizagem de um determinado conteúdo proposto pelo professor a seus alunos (NICOLA e PANIS, 2016). Esses mesmos autores também destacam que para a aula se tornar mais dinâmica e atrativa é importante que haja a utilização de recursos didáticos variados e devem ser ajustados a cada conteúdo programado com estratégias que visem a aprendizagem de forma mais significativa e proativa do aluno. $\mathrm{O}$ uso dos diversos recursos que pode contribuir com o aprendizado do aluno e o crescimento profissional do professor, possibilitando dessa forma maior interação professor-aluno e aluno-aluno (NICOLA e PANIS, 2016), podendo ser utilizados pelos professores, contribuindo para a aprendizagem e motivação de seus alunos.

Ressaltando a importância do uso dos recursos didáticos dentro da sala de aula, visando a prevenção dessas doenças, apresentando formas de higiene que ajudem na profilaxia (SANTOS et al., 2016), corroborando com NASCIMENTO (2019) que destaca a importância da utilização de recursos didáticos no ensino-aprendizagem de parasitologia, tornando-o mais dinâmico e significativo aos discentes.

Nesse sentido um meio alternativo para facilitar o ensino de parasitologia é a utilização de recursos didáticos tais como: modelos, maquetes, jogos e banners propiciando a compreensão dos aprendizes a respeito das doenças abordadas pela disciplina e que estão em constante diálogo com a realidade vivenciada pelos mesmos. Diante disso, o trabalho teve como principal objetivo a confecção e apresentação de materiais didáticos sobre amebíase por alunos do curso de licenciatura em Ciências Biológicas durante o I Simpósio Pinheirense de Biologia.

\section{METODOLOGIA}

Com base na metodologia proposta por SIMIELLI et al. (2017), produziu-se maquetes em biscuit abordando a morfologia do parasita, modo de transmissão, ciclo e órgãos acometidos pela doença. Vale ressaltar que as maquetes foram produzidas em 3D de modo que os deficientes visuais que por ali passavam pudessem ter uma maior interação com os materiais educativos disponibilizados. Houve o reaproveitamento de um manequim e desenharam-se os principais órgãos que a doença acomete, como: intestino grosso e fígado, para melhor exemplificar o ciclo da doença. E para facilitar a compreensão dos ouvintes sobre a espécie de Entamoeba histolytica que acomete o cérebro, confeccionou-se o órgão com isopor e biscuit para facilitar o entendimento de tal espécie, uma vez que ela se apresenta de forma mais grave (FIGURA 1). 
FIGURA 1: Mesa com material didático sobre Amebíase produzidos.

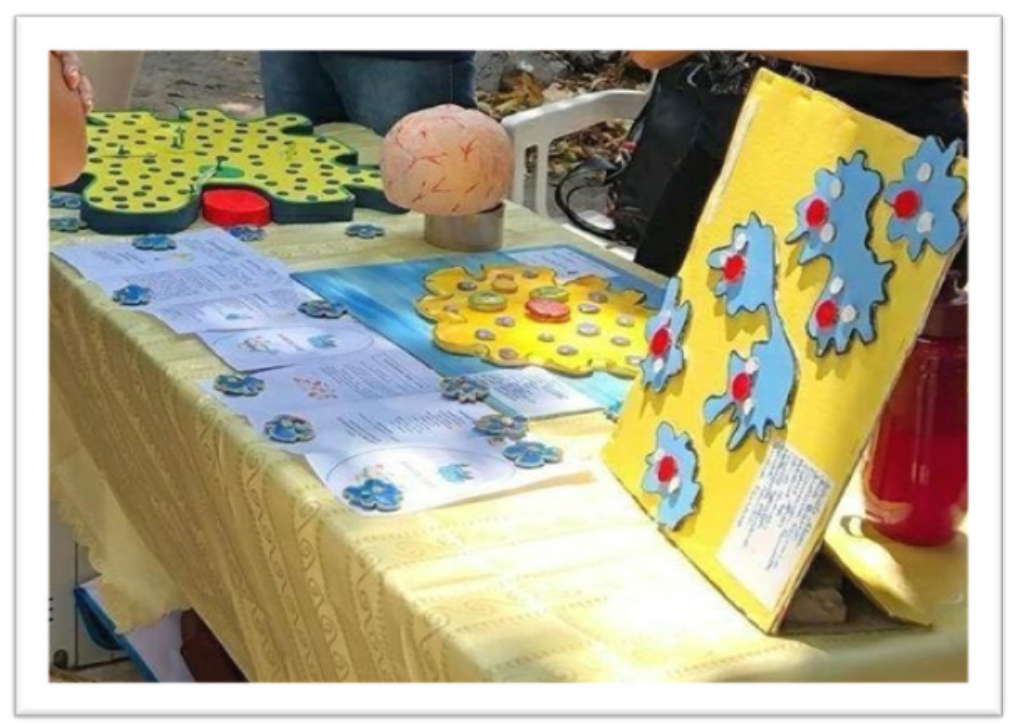

Fonte: Sarah Santos, 2019.

Além disso, foi produzido um banner dinâmico com imagens ilustrativas e textos curtos abordando tópicos como: O que é a doença; transmissão; ciclo da doença; sintomas; tratamento e prevenção de modo que o assunto pudesse ser rapidamente compreendido, na abordagem deu-se enfoque aos métodos de prevenção, uma vez que sendo realizados diminuem a probabilidade da infecção pelo parasita. E cartazes com ilustrações de medidas profiláticas, como: Lavar as mãos, fazer a boa higienização dos alimentos e mantê-los distantes de moscas e baratas que são considerados como vetores da doença. Além do mais produziu-se um jogo autoexplicativo onde os participantes iam montando e aprendendo sobre a estrutura morfológica do parasita e suas funções. 
FIGURA 2: Espaço montado durante o Simpósio Pinheirense de Biologia (SIMPHOBIO, 2019). Destancando Banner o informativo, o manequim reaproveitado demonstrando o intestino, modelos didáticos em biscuit.

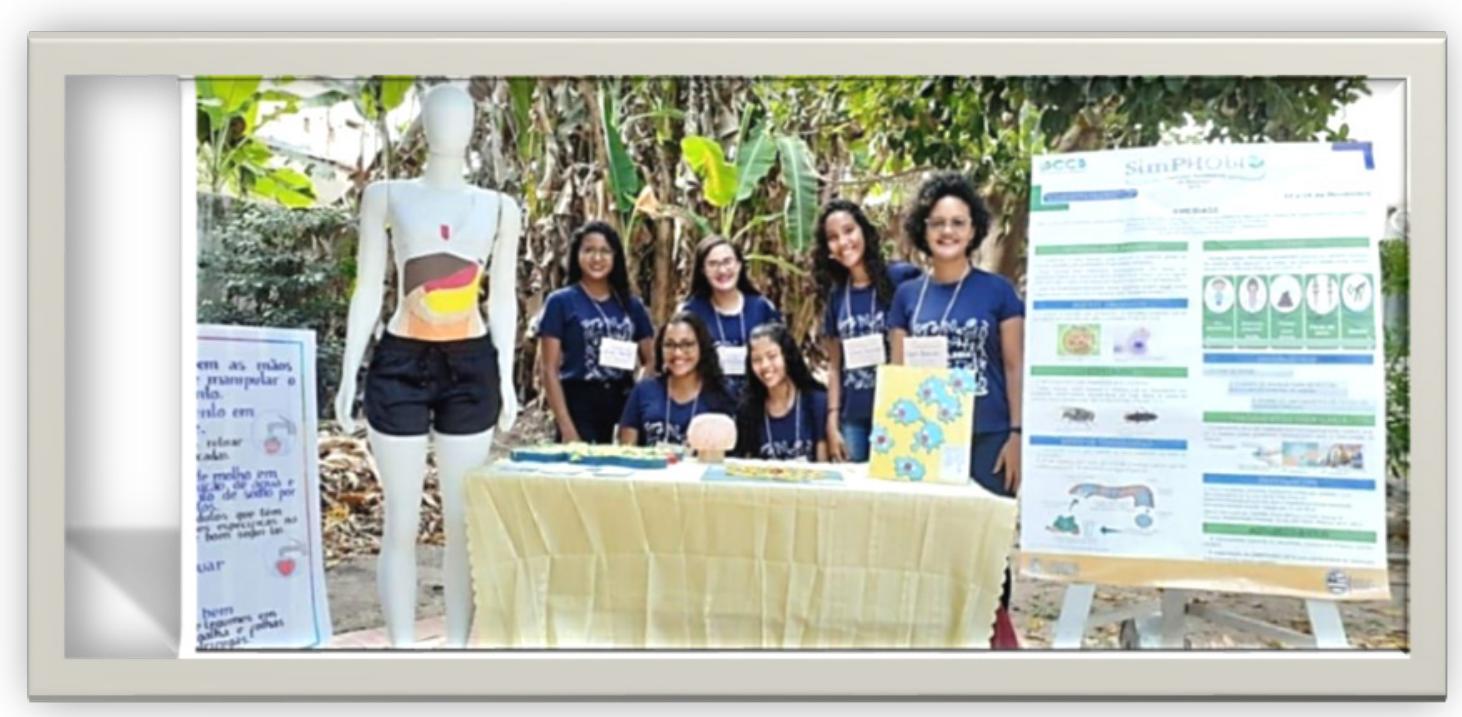

Fonte: Sarah Santos, 2019.

\section{RESULTADOS E DISCUSSÕES}

Os modelos didáticos segundo MORGAN e MORRISON (1999) são a representações de uma ideia, objeto, evento, processo ou sistema, criado para um propósito específico. Essas representações são confeccionadas a partir de material concreto, de estruturas ou partes de processos biológicos (MATOS et al., 2009). Sendo considerados como ferramentas didáticas capazes de sustentar a mediação entre ensino e aprendizagem, além de tornar as aulas de Ciências e Biologia mais dinâmicas. Em adição, Justi (2006) destacou que o aprendizado está relacionado tanto na construção do modelo didático, como em sua utilização, enfatizando que a teoria e a aprendizagem não se desvinculam do processo de produção, além da importância dos modelos como ferramenta de mediação e investigação.

Nicola e Paniz (2016) demonstraram os benefícios da utilização de diversos recursos didáticos na motivação, na transposição didática auxiliando na aprendizagem dos alunos, receptores do conhecimento. Corroborando com esse trabalho, onde percebemos que a apresentação do material produzido teve efeito positivo quanto a motivação, curiosidade, atenção e, consequentemente, no aprendizado sobre o tema proposto. No entanto, apesar dos benefícios muitos professores não utilizam recursos diferentes, talvez por medo do novo ou até mesmo por alguns padrões estabelecidos dentro do sistema educacional que não permitem o professor de utilizar tais recursos (NICOLA e PANIZ; 2016).

Durante a exposição do banner percebeu-se que grande maioria das pessoas que o observavam conseguiram assimilar com rapidez a abordagem do tema, fazendo assim com que fossem conduzidas a mesa expositiva levando-as a conhecerem a aprenderem mais sobre a doença. Anastasiou e Alves 
(2004) discutem a respeito da necessidade de aulas expositivas facilitando o processo de aprendizagem, pois, o professor deverá ser a ponte entre o conhecimento e o aluno, levando-os a questionamentos sobre o assunto desenvolvido, facilitando assim a produção de novos conhecimentos.

Durante a utilização de maquetes, foi perceptível a atenção dos que por ali passavam, levandoos a quererem saber mais sobre a doença abordada. Com a exposição do manequim e dos cartazes informativos ficou evidenciado que ainda há uma grande escassez de informações obtidas pelos alunos e comunidade em geral a respeito dos meios que os levam a contrair a doença, métodos de prevenção e os principais sintomas. Permitindo-nos inferir que grande maioria das pessoas que passaram pelo evento tem ou já tiveram alguma doença parasitária, porém, não sabiam como identificar os sintomas e fazer uso dos métodos preventivos.

Diversos participantes ficaram admirados com a exposição do cérebro e com a capacidade da doença em sua forma evolutiva de atingir o órgão podendo levar o homem que é seu hospedeiro definitivo a óbito. Para concluir a intervenção pedagógica, após a apresentação de todo o assunto referente à amebíase realizou-se a montagem do jogo auto-explicativo e percebeu-se que conforme a dinâmica ia acontecendo os ouvintes compreendiam melhor a estrutura morfológica do parasita e seu desenvolvimento dentro do organismo parasitado, ressaltando assim o que Passarelli (2012) argumenta a respeito da importância da ludicidade no processo de ensino-aprendizagem fazendo assim com que os ouvintes interajam com o material, tornando o assunto abordado mais dinâmico e de fácil compreensão.

Vale salientar que durante a apresentação das metodologias aqui mencionadas foram disponibilizadas cartilhas educativas para acompanhamento do assunto contendo informações relevantes a respeito da morfologia, habitat, transmissão, ciclo biológico, sintomas, profilaxia e curiosidades a respeito da amebíase.

\section{CONCLUSÃO}

Com base na aplicação dos materiais didáticos pode-se inferir que a utilização de diversas intervenções pedagógicas garantiu uma maior eficiência no processo de ensino-aprendizagem sobre o tema abordado, amebíase. Onde os diversos materiais ajudaram a prender a atenção dos ouvintes, tornando apresentação mais dinâmica e de fácil compreensão, pois foi despertado o interesse dos ouvintes pelo tema abordado. Desse modo fica evidente que materiais de baixo custo podem auxiliar no processo de aprendizagem não só dos discentes como também da comunidade geral, destacando assim a importância da educação em saúde com o objetivo de prevenir a contaminação da população pinheirense por doenças parasitárias, para que assim as pessoas que estiveram presentes no evento se tornem agentes disseminadores de conhecimento. 


\section{DECLARAÇÃO DE INTERESSES}

Nós, autores deste artigo, declaramos que não possuímos conflitos de interesses de ordem financeira, comercial, político, acadêmico e pessoal.

\section{REFERÊNCIAS}

ANASTASIOU, L. G. C.; ALVES, L. P. Estratégias de Ensinagem. 3. ed. Joinville: Univille, 2004. ALMEIDA, Amanda Andrade de; LEITE, Tailana Santana Alves. Entamoeba histolytica como causa da amebíase. Revista Saúde e Meio Ambiente - RESMA, Três Lagoas, v. 10, n. 1, p. 133-139, Jan./ Jul. 2020.

BASSO, Rita Maria Callegari et al. Evolução da prevalência de parasitoses intestinais em escolares em Caxias do Sul, RS. Revista da Sociedade Brasileira de Medicina Tropical, Caxias do Sul-RS, v. 41, 2008.

BARBOSA, Loeste de Arruda et. al. A educação em saúde como instrumento na prevenção de parasitoses. Revista Brasileira em Promoção da Saúde. Fortaleza, v. 22, n. 4, p. 272-278, Out./Dez. 2009. Disponível em: http://ojs.unifor.br/index.php/RBPS/article/view/1048. Acesso: 27 de nov. de 2020.

CORDEIRO, Thiago Guimarães Pires; MACEDO, Heloisa Werneck de. Amebíase. Revista de Patologia Tropical. [s.1], vol. 36 (2): 119-128. maio-ago. 2007.

JUSTI, Rosária. La Enseñanza de Ciencias Basada en la Elaboración de Modelos. Investigación Didáctica, Belo Horizonte- MG, p. 1-12, 2006.

LIMA, Jonatas Pereira de et al. Educação em Saúde: Ensino, Sensibilização e Prevenção de uma Parasitose Humana Negligenciada. Congresso Nacional de Educação, Rio Grande do Norte, p. 1-8, 2016.

LORENZETTI, L.; DELIZOICOV, D. Alfabetização Científica no Contexto das Séries Iniciais. Ensaio: Pesquisa em Educação em Ciências, v. 3, n. 1, 2001.

NASCIMENTO, Edilene da Cunha. Produção e utilização de jogos como estratégia didática para o ensino de Parasitologia na Educação Básica. Vitória de Santo Antão, 2019.

MATOS, Cysneiros et al. Utilização de Modelos Didáticos no Ensino de Entomologia. Revista de Biologia e Ciências da Terra, [s. l.], v. 9, ed. 1, p. 1-6, 2009.

MORRISON, M. y MORGAN, M. S. (1999). Models as medi- ating instruments, en Morgan, M.S. y Morrison, M. (eds.). Models as mediators, pp. 10-37. Cambridge: Cambridge University Press.

NICOLA, Jéssica Anese; PANIZ, Catiane Mazocco. A importância da utilização de diferentes recursos 
didáticos no ensino de ciências e biologia. InFor-Inovação e Formação, São Vicente do Sul - RS, p. 1-26, 2016.

PASSARELLI, L. M. G. Ensino e correção na produção de textos escolares. - 1. ed. - São Paulo: Telos, 2012.

SIMIELLI, M. E. R. et al. Do plano ao tridimensional: a maquete como recurso didático. Boletim Paulista de Geografia, n. 70, p. 5-22, 2017.

TESORI, Suzana Patricia. Produção de materiais pedagógicos como estratégia de ensino de Biologia. Trabalho de Curso, Abelardo Luz/SC, p. 1-22, 2018. 


\section{ÍNDICE REMISSIVO}

\section{Símbolos}

$1^{\mathrm{a}}$ e $2^{\mathrm{a}}$ Lei de Mendel 114,116

A

alelos 98, 99, 100, 101, 102, 105, 107, 108, 110, 117, 118, 119, 121, 122, 123, 124

alfabetização científica 42, 44, 51, 61, 62

alginato $42,44,45,46,48,52,53,55,56,57,59,64$

alginato de sódio 42, 44, 45, 46, 53, 56, 57

ambiente escolar $13,29,53,55,58,89$

animais nativos $76,80,84$

animais vertebrados $76,77,78,83$

animais vertebrados e o ser humano 76

Aprendizagem 18, 65, 67, 112

aprendizagem de biologia 114

atividade experimental $44,45,46,53,55,56,58,62$

atividades remotas 11,15

aulas de biologia 28

aulas presenciais $11,15,17,89,90$

aulas remotas 11,13

a vida em sociedade 127

B

Biologia $6,11,14,15,16,17,20,22,24,26,27,37,40,41,63,85,86,88,100,110,112,115,116,124,125,133$

biologia celular 114,115

botânica 65,70

C

campo da ecologia 65,69

campo da nanotecnologia 53,62

caráter histórico e dinâmico da ciência $\square 28,36,37$

carreadores de fármacos $42,43,44$

cátions bivalentes 53,55

ciclo da doença $20,22,23$

ciência como atividade coletiva $\square 28,36,37,38$

Ciências $11,12,14,15,16,18,22,24,26,40,41,43,44,48,50,62,65,67,75,87,91,110,111,112,117,125,137$ 
citologia $37,65,70$

compreensão e fixação das informações 19

comunidade $20,22,25,76,77,78$

conceito de homofobia 127,134

concentração nos estudos 11, 15

conexão de internet 11

conhecimentos da genética 114

consciência biológica 76, 84

construção de cordéis 65,67

conteúdo didático 11,12

conteúdo informativo 20

cromossomos $98,99,100,101,102,104,105,106,108,109,110,114,115,117,118,119,120,121,122,123,124$

$\mathbf{D}$

Dança dos Cromossomos 114, 116

déficit informativo 19

didáticas alternativas 98

dificuldade de entender os conceitos 11

dificuldade na compreensão 97

Dificuldades de aprendizagem 11, 111, 125

dificuldades do aprendizado 11

dinâmica das aulas 87,88

disciplina de genética 97, 115

dispositivo eletrônico 11, 16

disseminação de conhecimento 20, 21

diversidade $71,77,127,132$

doenças negligenciadas 19

dominância completa 98, 100

E

Educação básica 11

educação sexual 127,135

Enfrentamento das visões ingênuas sobre a ciência 28

ensino da hereditariedade 97

ensino de ciências $27,28,32,40,42,51,96,125$ 
ensino de parasitologia 19,22

ensino remoto $11,13,14,17,87,88,89,90,91,92,96$

entendimento sobre a amebíase 20

esferas de alginato $42,45,46,47,48,53,56,57,58,59,60$

espécie humana 77, 101

espermatozoides 98, 100, 102, 107, 108, 109

Estratégias 26, 63, 65

estudantes de biologia 98

Estudos Culturais em Educação 127, 128

estudos histórico-filosóficos 28

etapa experimental 53

F

falta de reflexão sobre a $\mathrm{NdC} 28$

família 76, 80, 136

farmacocinética 42,50

fármacos $42,50,55$

fechamento das escolas 11,12

fenômeno biológico 101, 108, 113, 115

fenômenos biológicos 114

fenótipo 98, 100, 103, 108, 124

ferramenta didática $65,67,114$

ferramenta didática adjuvante 65

fisiologia humana 87, 91, 94

formação dos gametas 114, 117, 118, 119, 121, 124

formato de aprendizagem 11

formulação convencional 42

fusos meióticos 114, 120, 122

G

genótipo 98, 100, 101, 102, 124

graduação nas áreas biológicas 98

H

hereditariedade $98,99,101,110,111,112,113,114,115,125,133$

homem e natureza $76,77,82,84$ 
homem e natureza/vertebrados 76

homem primitivo 76,77

homossexualidade 127, 128, 129, 130, 131, 132, 133, 134, 135, 136, 137

I

Inclusão 11

inteligência cinestésico-corporal 114, 116

interdisciplinaridade 42,50

interesse em aprender 87, 95

J

jovens em idade escolar 11

$\mathbf{L}$

Leis de Mendel 110, 113, 114, 115, 125

Ligação Gênica 114, 116, 121, 123, 125

linguagem e conceitos complexos 19,21

lipossomas 42,44

Literatura $65,74,75$

literatura de cordel $65,66,67,68,73,74,75$

livro paradidático de sexualidade 127, 128, 129, 131, 132

livros didáticos $28,31,32,33,34,35,36,38,39,41,48$

livros didáticos de biologia $28,31,32,34,35,36,38,41$

livros didáticos de ciências/biologia 28

M

manifestações culturais 65,66

materiais didáticos 14, 20, 22, 25, 31, 52, 62, 63

materiais poliméricos 53

mecanismos biológicos 113

medicamentos $42,43,44,58$

meio ambiente $65,69,77,78$

meiose $71,101,114,116,117,118,119,124,125$

Mentimeter 87, 88, 89, 92

Metodologias 14, 18, 65, 95, 96

metodologias ativas $14,87,89,90,91,94,96$

métodos profiláticos 20 
microestruturas 53,55

minimizar prejuízos 11

Ministério da Educação 11, 88

Ministério da Saúde 11

modelos didáticos 24, 98,100

modo de transmissão 20,22

mudanças no âmbito educacional 87, 88

$\mathbf{N}$

nano- e micropartículas 42

nanosistemas 42

nanotecnologia $43,50,53,54,55,56,57,58,61,62,63,64$

nanotecnologia e suas aplicações 54, 57, 62

natureza da ciência (NdC) 28, 29

novo coronavírus 87,88

O

oficinas de versificação 65

$\mathbf{P}$

Padlet 87, 88, 89, 92, 93

padrões de herança 98, 100, 101, 103, 110, 111

pandemia $11,12,14,17,18,87,88,89,96$

pandemia do COVID-19 11, 14

panfletos informativos 20

participação ativa $14,62,87,91,92,93,94,95$

participação mais efetiva 43, 44, 51, 62

Pecha Kucha 87, 88, 89, 90, 91, 92, 93

perspectiva cidadã 127

perspectiva sociocultural 127

plataformas digitais 11,15

polissacarídeo natural 44, 53, 55

poluição $55,65,69,70,82$

povo nordestino 65

principais dificuldades 11

problematização 28, 129, 134 
processo da fecundação 98,101

processo de ensino-aprendizagem 20, 25, 39, 52, 87, 100, 115, 124

professor de biologia 114,125

protozoário Entamoeba histolytica 20, 21

R

recursos didáticos alternativos 19,100

relação ecossistêmica 76,80

responsabilidade com o ambiente 76,84

$\mathbf{S}$

segurança $11,12,81$

simulações 98, 123

sobrevivência $76,78,79,80,83$

subsistência $76,77,79,80,82,83,84$

sub terapêutica 42

suspensão das aulas presenciais 11

sustentabilidade $65,69,71$

T

Tecnologias Digitais de Informação e Comunicação (TDICs) 87, 89

teoria celular $28,31,32,37,38$

terapêutica 42,49

Trilha da aprendizagem $87,89,90,92$

$\mathrm{U}$

uso de jogos 98

V

vírus SARS-CoV-2 11,12

$\mathbf{Z}$

zoologia 65,70 
